\title{
Ultrathin and stable AgAu alloy nanowires
}

\author{
Jianbo $\mathrm{Wu}^{1}$, Yung-Tin $\mathrm{Pan}^{1}$, Dong $\mathrm{Su}^{2}$ and Hong Yang ${ }^{1 *}$
}

\begin{abstract}
Stable sub-two nanometer AgAu nanowires were synthesized using a $\mathrm{CO}$-mediated gas reducing agent in liquid solution (GRAILS) method. These AgAu nanowires are stable, and do not readily break into short nanorods upon heating or electron beam irradiation. The surfaces along the longitudinal direction of the AgAu nanowires were largely bounded by the stable $\{111\}$ facets. This structure differs from those ultrathin Au nanowires made without the use of $\mathrm{CO}$ gas, which were bounded by the $\{100\}$ facets and unstable under e-beam irradiation. These differences in structure and composition lead to enhanced stability.
\end{abstract}

One-dimensional metal nanostructures have interesting optical, electronic, catalytic, and transport properties [1-8]. Among the different metals, Au and Ag nanowires and nanorods show strong absorption in the range from visible to near infra-red (NIR) region. They also possess excellent thermal and electrical conductivity, which are useful in a variety of applications, such as interconnects in electronic system $[9,10]$, optical devices $[11,12]$, spectroscopic enhancers [13-15], and electrochemical [16-19] and biological sensors [20]. For instance, electronics in an integrated circuit need to work properly above $100^{\circ} \mathrm{C}$, thus the suitable metal nanowires have to be electrically highly conductive and thermally stable. For this purpose, Au nanowires are used despite the high cost. Ag has been considered as an alternative due to its relative cheaper price and high electrical $\left(6.3 \times 10^{7} \mathrm{~S} \mathrm{~m}^{-1}\right)$ and thermal $\left(429 \mathrm{~W} \mathrm{~m}^{-1} \mathrm{~K}^{-1}\right)$ conductivity [21]. In recent years, a large amount of flexible transparent conductive film was made based on Ag nanowires [22]. Thin wires are desirable in terms of ductile property. In addition, $\mathrm{Ag}$ and $\mathrm{Au}$ nanomaterials also attract much attention for their applications in photo-thermal cancer therapy and near infrared light, which offers deeper penetration than the visible light [23]. The unique localized surface plasmon resonance (LSPR) produced by $\mathrm{Ag}$ and $\mathrm{Au}$ nanostructures, especially $\mathrm{Ag}$, is strong and thus also of great importance for bio-imaging [24-26]. However, Ag is prompt to be oxidized by oxygen in air or other oxidant, thus Ag-Au alloy, which is more stable than either pure Au or Ag [27], should have the ad- vantages in this regard. The synthesis of $\mathrm{AgAu}$ nanowires, however, has been unsuccessful, especially when the diameter approaches $2 \mathrm{~nm}$, at which point metal nanowires become very ductile [28].

One-dimensional metal nanomaterials are typically made using surfactant-mediated growth method [29], although template approach and oriented attachment are also used [30,31]. These methods often yield metal nanorods with diameter larger than $5 \mathrm{~nm}$. A liquid crystal mesophase-assisted approach was developed recently for producing ultrathin $\mathrm{Au}$ nanowires $(<2-3 \mathrm{~nm})$ [32-35]. These ultrathin $\mathrm{Au}$ nanowires, however, were not stable and readily broken into short nanorods even under moderate electron beam (e-beam) irradiation, making them practically impossible to be utilized otherwise intriguing plasmon properties from the longitudinal component of these metal nanowires [36,37]. Metal nanostructures are known to have different thermal properties from their bulk counterparts due to surface energy difference [38]. The size-dependent thermal stability of Au nanoparticles including rods has been studied experimentally using ultrafast lasers [39-42], and by theoretical modeling [43-45]. In the case of ultrafine $\mathrm{Au}_{38}$ nanoparticles, the melting point lowers to $180^{\circ} \mathrm{C}[46,47]$. Nanowires however can undergo various structural changes at temperatures lower than the estimated melting points for spherical nanoparticles due to the Rayleigh instability [48]. In this case, the driving force for the structural change is the chemical potential gradient (capillarity or surface tension) induced by a perturbation in the radial direction of the nanowires [48].

Recently, thin Au nanowires were made by using carbon monoxide at a low flow rate [49]. Unlike the Au nanowires made by using mesophase-template alone, these $\mathrm{Au}$ nanowires seem to be stable under e-beam irradiation and no short rods were shown in the TEM micrograph. This result suggests that structural stability of nanowires could be closely related to the surface tensions of the nanowires, and both facet and surface capping ligands may be important. Herein, we report the synthesis of high aspect ratio AgAu

\footnotetext{
${ }^{1}$ Department of Chemical and Biomolecular Engineering, University of Illinois at Urbana-Champaign, Urbana, Illinois 61801, USA

${ }^{2}$ Brookhaven National Laboratory, Center for Functional Nanomaterials, Upton, NY 11973-5000, USA

*Corresponding author (email: hy66@illinois.edu)
} 
nanowires with an average diameter of $\sim 2 \mathrm{~nm}$ via a low temperature solution phase synthesis assisted by CO gas and present a study on the stability of these alloy nanowires under e-beam irradiation and at elevated temperatures.

Fig. 1a shows representative transmission electron microscopy (TEM) micrographs of AgAu nanowires obtained from a mixture of $0.05 \mathrm{mmol}$ of $\mathrm{HAuCl}_{4}$ and $0.05 \mathrm{mmol}$ of silver trifluoroacetate (AgTFA) in $5 \mathrm{~mL}$ of oleylamine (OAm) and $5 \mathrm{~mL}$ of oleic acid (OAc). The reaction took place at $60^{\circ} \mathrm{C}$ for $1 \mathrm{~h}$ using the $\mathrm{CO}$-assisted gas reducing agent in liquid solution (GRAILS) method [50]. The population of nanowires was about $90 \%$ and had a high aspect ratio of $\sim 100$, with an average diameter of $2.1 \pm 0.3 \mathrm{~nm}$ and length of $200 \mathrm{~nm}$ (Fig. 1a inset and Fig. S1). Nanoparticles with diameter of $\sim 6 \mathrm{~nm}$ made up the rest. High $\mathrm{CO}$ flow rate $(190 \mathrm{sccm})$ and elevated temperature $\left(60^{\circ} \mathrm{C}\right)$ are required to produce $\mathrm{AgAu}$ nanowires. Only $\mathrm{Au}$ nanowires were obtained either at a lower CO flow rate or a temperature less than $45^{\circ} \mathrm{C}$.

The sides of these nanowires are largely enclosed by the $\{111\}$ surfaces, as shown in Fig. 1b. The $d$-spacing of these planes is $0.23 \mathrm{~nm}$, which indicates that the sides of nanowires are not enclosed by $\{100\}$ surfaces, nor are the single crystalline nanowires that grow along the $\langle 100\rangle$ direction and are bounded with $\{100\}$ and $\{110\}$ facets on the side, as in the cases of previous reports [51]. The tips of AgAu nanowires could have the twinned structures bounded by the $\{111\}$ surfaces (Fig. S2) [52]. Formation of the $\{111\}$ surface-enclosed nanowires could be ascribed to facet-dependent preferential adsorption of $\mathrm{CO}$ gas on metal surfaces $[16,50,53,54]$. In the absence of $\mathrm{CO}, \mathrm{Au}$ nanowires grew along $\langle 111\rangle$ direction, rather than bounded by $\{111\}$ surfaces [32,33]. Fig. 1c shows a high-angle annular dark-field scanning transmission electron microscopy (HAADFSTEM) image of the nanowire, and Fig. 1d is the corresponding line scan profiles of $\mathrm{Au}$ and Ag elements across a nanowire based on the energy-dispersive X-ray (EDX) analysis. The EDX linear scan shows that both Au and Ag elements are fairly evenly distributed across the nanowire (Fig. 1d). The shapes of EDX line scans for the two elements are similar and have a peak in the central region, indicating the one-dimensional structure is wire, not ribbon. EDX analysis further shows that the atomic ratio between $\mathrm{Au}$ and $\mathrm{Ag}$ is about 1:1, close to the feeding ratio between the two metals in the precursors (Fig. S3).

OAm and OAc were needed to form a liquid crystal
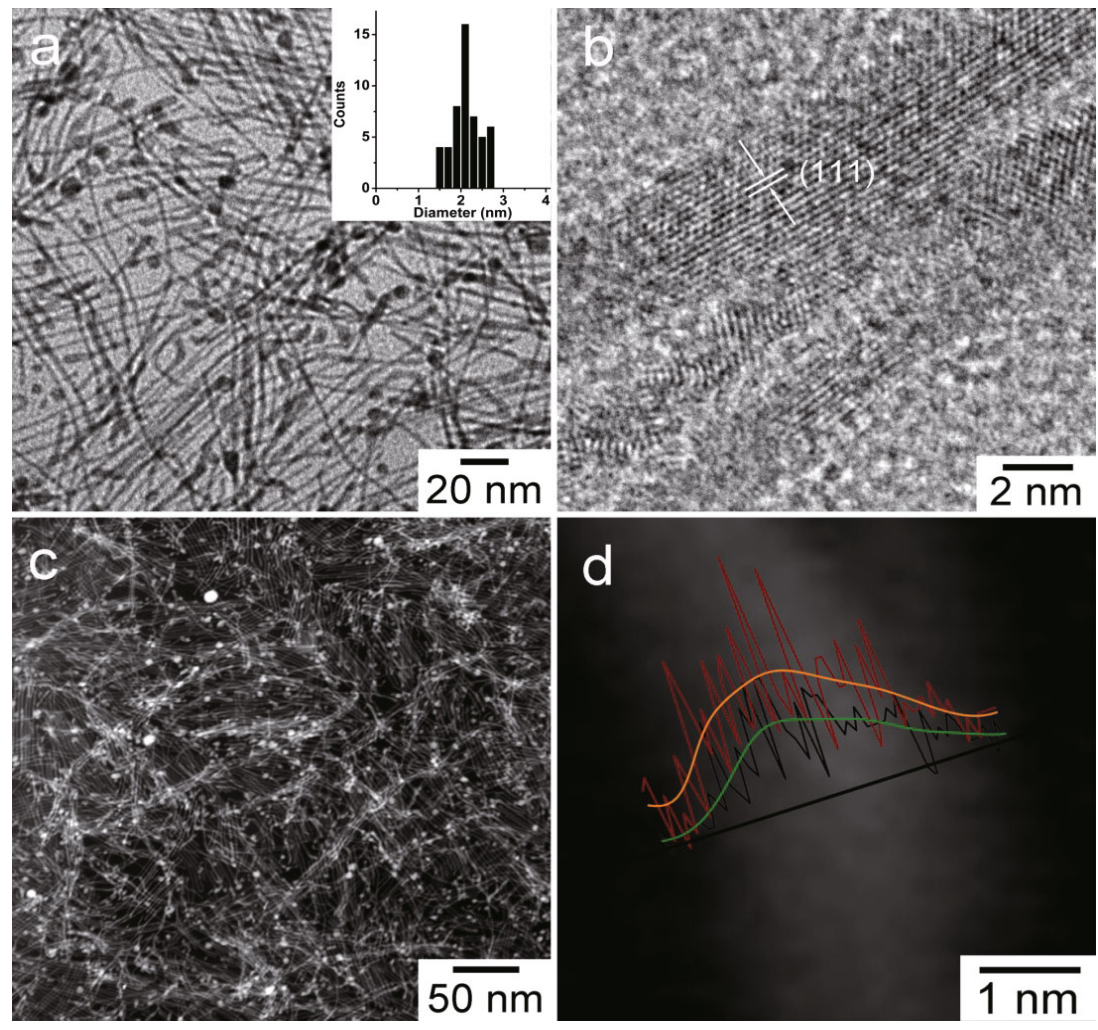

Figure 1 (a) Low and (b) high magnification TEM micrographs, (c) HAADF-STEM image, and (d) corresponding EDX line scans of Au (gold line) and $\mathrm{Ag}$ elements (green line) of $\mathrm{AgAu}$ nanowires. The size distribution is shown as the inset in (a). 
phase and to assist the binding of metal ions to facilitate the growth of AgAu nanowires [32,33]. The aspect ratio of the nanowires was controllable by adjusting the OAm/OAc ratio. When $5 \mathrm{~mL}$ of OAm and $5 \mathrm{~mL}$ of OAc were used, nanowires with a length of 100-150 nm were obtained (Fig. S1). The length of the nanowires shortened to $50-80 \mathrm{~nm}$ if the volumetric ratio between OAm and OAc increased to 7:3 (Fig. S4a). $7 \mathrm{~nm}$ nanoparticles were obtained when this ratio was changed to 3:7 (Fig. S4b). Reaction temperature is critical for the morphology of the nanowires. Fig. S5 shows the nanowires were around $100 \mathrm{~nm}$ long and 2.2 $\pm 0.4 \mathrm{~nm}$ in diameter when the reaction temperature was $45^{\circ} \mathrm{C}$. These nanowires were shorter than those obtained at $60^{\circ} \mathrm{C}$ with the same reaction time period of $30 \mathrm{~min}$. The $\mathrm{Au}: \mathrm{Ag}$ atomic ratio of the final product was close to $1: 1$, which was the feeding ratio between the two metal precursors, indicating that $\mathrm{Au}$ and $\mathrm{Ag}$ precursors could almost completely be reduced even at $45^{\circ} \mathrm{C}$ (Fig. S6). When the reaction temperature was lowered to $25^{\circ} \mathrm{C}$, the nanowires formed was made of mostly Au (Figs S7a and S8). The reduction of $\mathrm{Ag}$ precursor appeared to be slower than $\mathrm{Au}$ at this temperature. Noticeably, these $\mathrm{Au}$ nanowires had a similar average diameter of $2.2 \pm 0.3 \mathrm{~nm}$, but an average length of 50-80 $\mathrm{nm}$. Moreover, only Au nanowire could be obtained at $60^{\circ} \mathrm{C}$, if the flow rate of $\mathrm{CO}$ gas was lower than $100 \mathrm{sccm}$. The as-made Au nanowires in presence of $\mathrm{CO}$ were also enclosed predominantly by $\{111\}$ due to the surface selective adsorption of CO (Fig. S7b) [50,53-55]. These results indicate that $\mathrm{AgAu}$ nanowires can only be synthesized at elevated temperature and with a sufficiently high flow rate of $\mathrm{CO}$ gas.

The stability of $\{111\}$ facet-enclosed AgAu nanowires and $\{100\}$ facet-enclosed $\mathrm{Au}$ nanowires were investigated using field emission TEM (FEI Tecnai F20) at an accelerating voltage of $200 \mathrm{kV}$. The AgAu nanowires were stable under the e-beam for an extensive period of time ( $30 \mathrm{~min})$. In contrast, the sub- $2 \mathrm{~nm}$ Au nanowires made without $\mathrm{CO}$ gas were highly susceptible to e-beam damage. They broke up within 1-5 min exposure under e-beam irradiation at a dose level of $8 \mathrm{pA} \mathrm{cm}^{-2}$, even if they were embedded in the thick surfactant media (Fig. S9). These ultrathin Au nanowires broke into short rods in about $5 \mathrm{~s}$ under the e-beam irradiation if there was no imbedded media (Fig. S10). Thick OAm and OAc surfactant media could in part prevent the Au nanowires from breaking up rapidly under the e-beam irradiation. Au nanowires less than $2 \mathrm{~nm}$ in diameter and with the longitudinal direction along $\langle 111\rangle$ were previously shown to readily break into short rods upon e-beam irradiation [32,33]. Those ultrathin Au nanowires primarily grew along $\langle 111\rangle$ direction and bounded by $\{100\}$ facets on the sides when CO gas was not used $[33,56]$. Au nanowires made under those conditions were unstable and could result in shape transformation into shorter but thicker nanorods due to Rayleigh instability $[43,48,52]$. In comparison, both $\mathrm{Au}$ and AgAu nanowires generated under CO gas were predominately enclosed by $\{111\}$ surfaces and stable under the e-beam irradiation.

The thermal stability of $\{111\}$ surface-enclosed AgAu and $\mathrm{Au}$ nanowires was investigated with the heat treatment. AgAu nanowires remained largely intact after heating at $60^{\circ} \mathrm{C}$ in chloroform for $1 \mathrm{~h}$, though the diameter increased slightly to $2.5 \pm 0.4 \mathrm{~nm}$, which is close to eight unit cell length (Fig. 2a). The longitudinal surfaces were still primarily bounded by $\{111\}$ facets (Fig. 2b). The length of

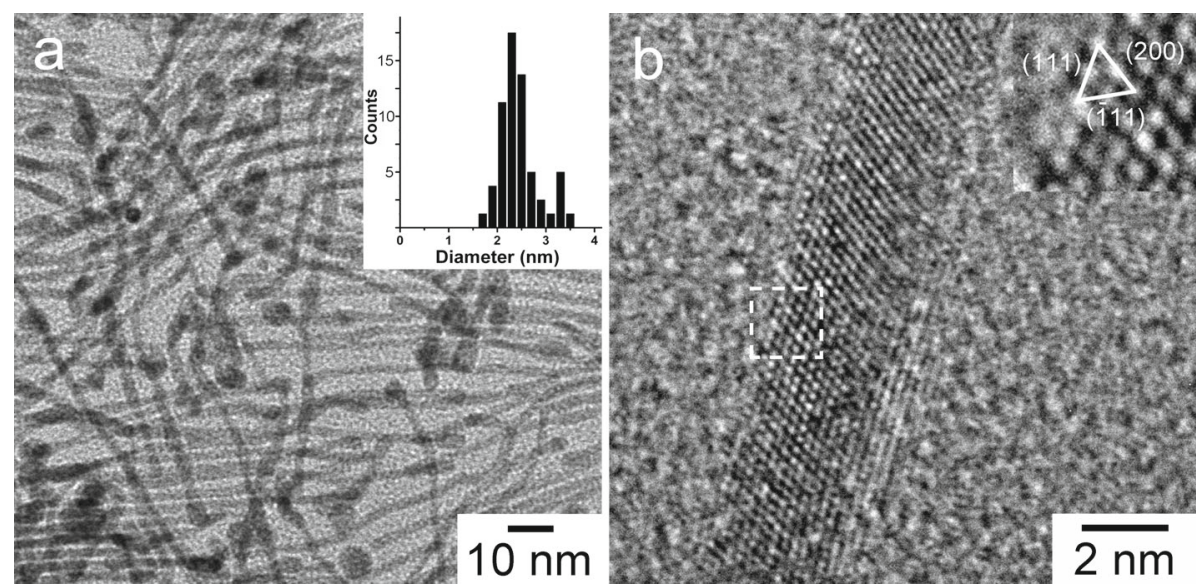

Figure 2 (a) High and (b) low magnificent TEM micrographs of AgAu nanowires after annealing at $60^{\circ} \mathrm{C}$. Inset shows the size distribution of diameter of the nanowires. 
Au nanowires made by using $\mathrm{CO}$ gas was largely preserved after the heat treatment at $60^{\circ} \mathrm{C}$ in chloroform for $1 \mathrm{~h}$, although some nanowires show uneven contrast along the longitudinal direction within the wire (Fig. S11). This unevenness is likely caused by the Rayleigh instability [48].

Fig. 3 shows the TEM micrographs of $\{100\}$ facet-enclosed $\mathrm{Au}$ nanowires. After thermal treatment at $60^{\circ} \mathrm{C}$ in chloroform for $1 \mathrm{~h}$, these Au nanowires, which were hundreds of nanometers long originally, broke into $20-50 \mathrm{~nm}$ short rods. These nanowires showed both single-crystalline and polycrystalline features and were enclosed by $\{200\}$ and $\{110\}$ facets. Noticeably, the latter two metal surfaces are generally less stable than $\{111\}$ surfaces [57]. Reconstruction of these thin nanowires occurred far below the melting temperature of Au. Upon heating, such nanowires underwent a shape transformation to either short and widened rods or spherical nanoparticles (Fig. 3b). Figs 3c and d show high resolution TEM micrographs of these $\mathrm{Au}$ nanowires having the reconstruction at both center and tip regions after annealing. Two sets of lattices separated by a planar defect were observed within the nanowire. One is projected along $\langle 111\rangle$ direction, showing (200) surface that has a $d$-spacing of $0.20 \mathrm{~nm}$. The other is projected along $\langle 110\rangle$ direction, with $d$-spacing of $0.234,0.200$ and 0.144 nm for (111), (200) and (110) planes, respectively. Fig. 3d shows the TEM micrograph of the surface reconstruction at the tip region. The atoms at the outer layer turned into $\{111\}$ surface structures, while those in the interior kept the same structure in $\{200\}$ lattice. This structural change could be driven by the requirement for minimizing the surface energy. The tips of $\mathrm{Au}$ nanowires have high surface energy with large curvature and the surface atoms are prompted to the reorganization from the high energy $\{110\}$ surfaces to the low energy $\{111\}$ surfaces.

The thermal stability of AgAu nanowires was also studied using in situ variable temperature TEM (Fig. 4). These ultrathin alloy nanowires were stable under e-beam irradiation at an accelerated voltage of $200 \mathrm{kV}$. Some started to break around $50^{\circ} \mathrm{C}$ at the narrow regions of the nanowires. About $10 \%$ of the nanowires (i.e., 5 out of 50 in the micrograph) broke into short rods when the temperature rose to the range of $60-100^{\circ} \mathrm{C}$. The tips of the broken nanowires had spherical shapes because of the reconstruction through the Rayleigh process (Fig. S12) [48]. The variation of local

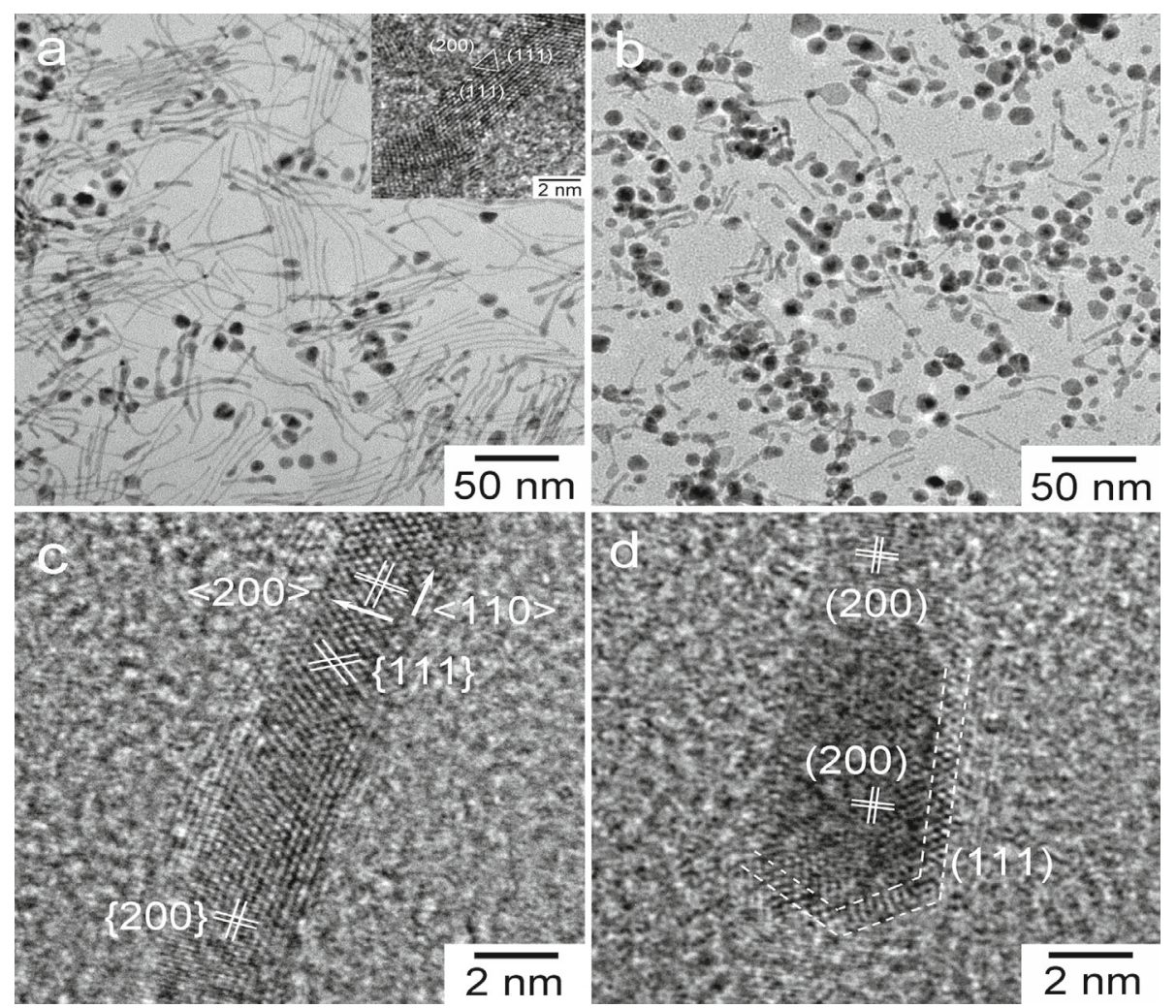

Figure 3 TEM micrographs showing the morphology and structure changes of Au nanowires (a) before and (b, c, d) after a thermal treatment at $60^{\circ} \mathrm{C}$ in chloroform for $30 \mathrm{~min}$. Inset of (a) shows the lattices of Au nanowires in HR-TEM micrograph. These Au nanowires were made without the use of CO gas. 

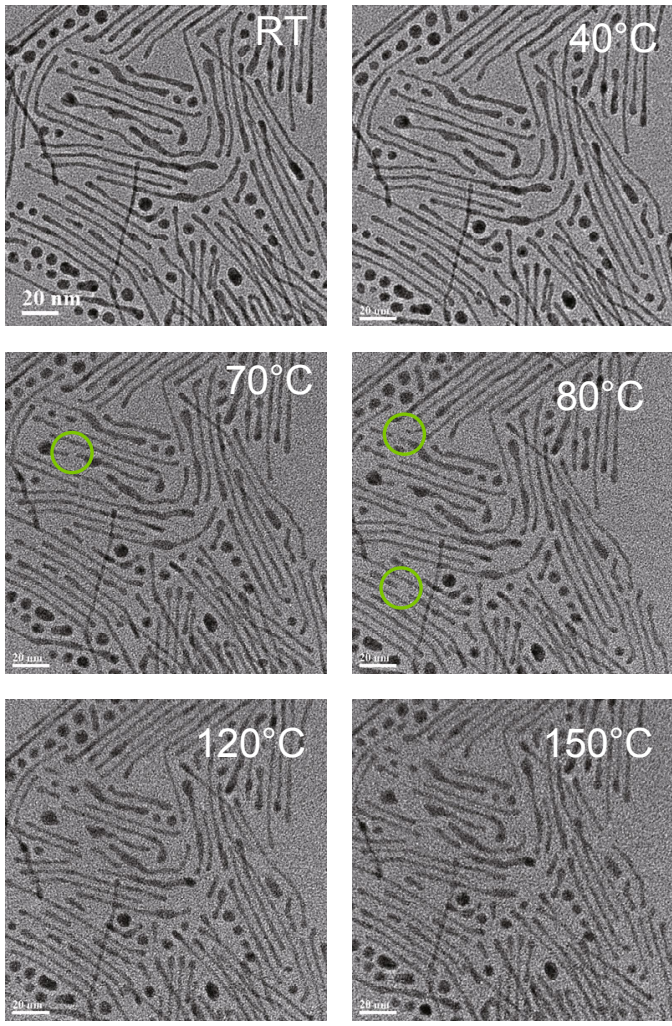
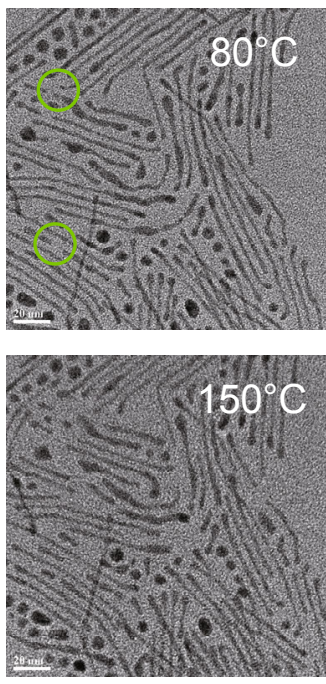

Start to break
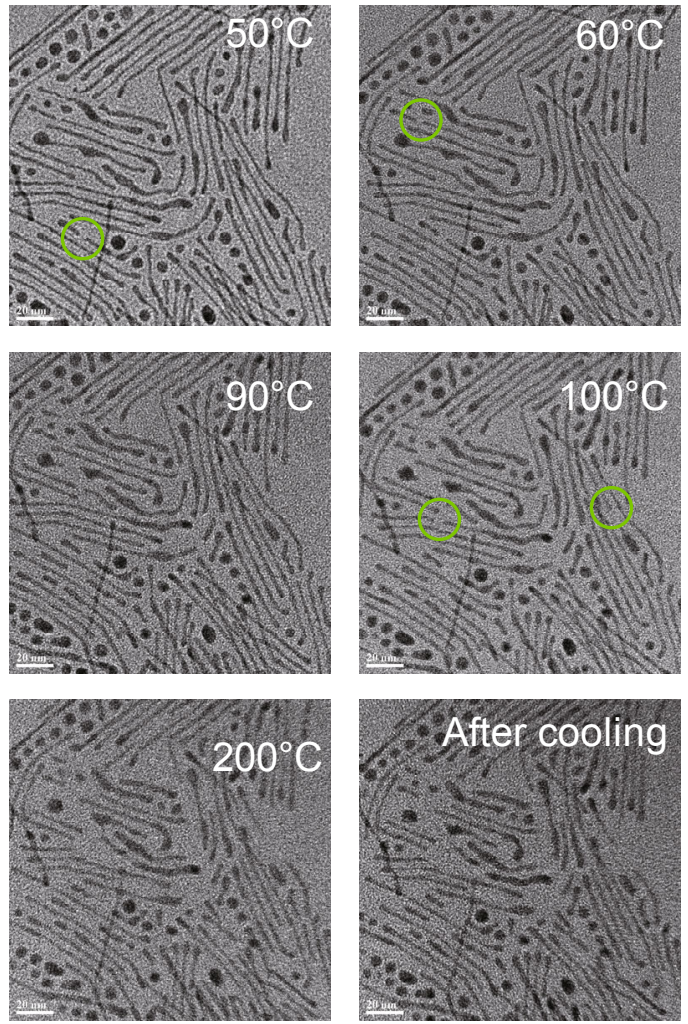

Figure 4 In situ variable-temperature TEM study of morphological changes of AgAu nanowires during heat treatment at different temperatures.

composition might contribute to the thermal instability of nanowires. The rest of alloy nanowires were fairly stable even when the temperature reached $200^{\circ} \mathrm{C}$. These nanowires apparently could tolerate large strain without morphological changes during the cooling process. The convergent evident from the TEM study indicates overall the composition of the wires is homogeneous and no severe phase separation was observed. If there was large phase separation, the local strain would cause the changes in morphology and contrast which were not observed in the TEM images.

Fig. 5 shows the UV-vis-NIR absorption spectra for ultrathin $\mathrm{AgAu}$ alloy nanowires. As a comparison, $\mathrm{Au}$ nanowires made without $\mathrm{CO}$ gas were also examined under the same condition. Both $\mathrm{AgAu}$ and $\mathrm{Au}$ nanowires show two LSPRs: one around $500 \mathrm{~nm}$ associated with the transverse resonance and the other with longitudinal resonance at $2900 \mathrm{~nm}[16,58,59]$. The AgAu nanowires exhibited essentially the same kind of UV-vis absorption spectra after the heat treatment between 60 and $100^{\circ} \mathrm{C}$. This observation indicates that most of the $\mathrm{AgAu}$ alloy nanowires should be thermally stable within this temperature range.
On the contrary, after annealing at $60^{\circ} \mathrm{C}$ the longitudinal resonance of $\mathrm{Au}$ nanowires blue-shifted by $\sim 300 \mathrm{~nm}$, and after the treatment at $100^{\circ} \mathrm{C}$, a broad band between 1500-2800 $\mathrm{nm}$ appeared (Fig. S13), indicating the coexistence of $\mathrm{Au}$ nanostructures with huge variations in their dimensions. The background absorption due to the solvent and surfactants can be ruled out (Fig. S14). Thus, the blue shift in the NIR region could be attributed to the fragments of the broken nanowires.

In summary, we developed a $\mathrm{CO}$-assisted synthesis of ultrathin, high aspect ratio AgAu alloy nanowires. These $\mathrm{AgAu}$ nanowires, which are largely bounded by $\{111\}$ planes along the longitudinal surfaces, are much more stable than ultrathin Au nanowires bounded by $\{100\}$ facets made without the use of $\mathrm{CO}$ gas. These ultrathin AgAu nanowires possess stable transverse resonance in the visible and longitudinal resonance in the NIR region. Besides a new synthetic method for making AgAu alloy nanowires, the discovery of facet-dependent stability in ultrathin metal nanowires is important for the design of new classes of stable low dimensional nanomaterials. 

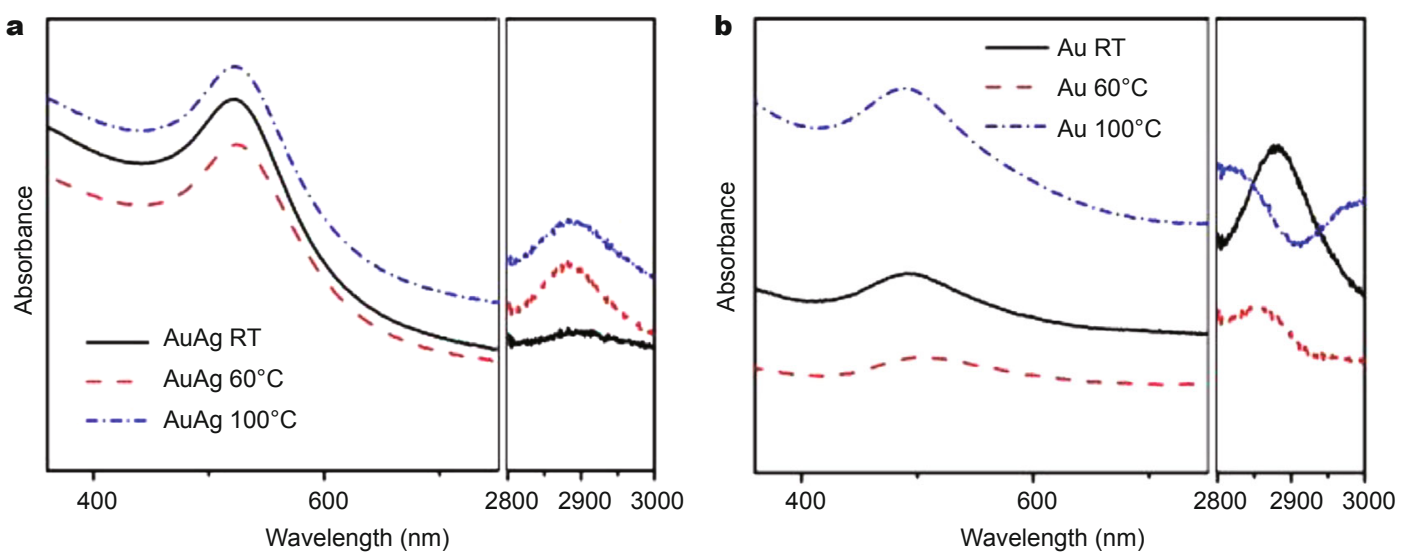

Figure 5 UV-vis-NIR absorption spectra for ultrathin (a) AgAu and (b) Au nanowires with and without thermal treatments.

Received 11 June 2015; accepted 8 July 2015; published online 11 August 2015

1 Huang $\mathrm{MH}$, Mao S, Feick H, et al. Room-temperature ultraviolet nanowire nanolasers. Science, 2001, 292: 1897-1899

2 Xia YN, Yang PD, Sun YG, et al. One-dimensional nanostructures: synthesis, characterization, and applications. Adv Mater, 2003, 15: 353-389

3 Li Y, Qian F, Xiang J, et al. Nanowire electronic and optoelectronic devices. Mater Today, 2006, 9: 18-27

4 Josell D, Brongersma SH, Tokei Z. Size-dependent resistivity in nanoscale interconnects. Ann Rev Mater Res, 2009, 39: 231-254

5 Shi P, Bohn PW. Electrochemical control of stability and restructuring dynamics in $\mathrm{Au}-\mathrm{Ag}-\mathrm{Au}$ and $\mathrm{Au}-\mathrm{Cu}-\mathrm{Au}$ bimetallic atom-scale junctions. ACS Nano, 2010, 4: 2946-2954

6 Lal S, Hafner JH, Halas NJ, et al. Noble metal nanowires: from plasmon waveguides to passive and active devices. Acc Chem Res, 2012, 45: 1887-1895

7 Ji J, Zhou Z, Yang X, et al. One-dimensional nano-interconnection formation. Small, 2013, 9: 3014-3029

8 Chen C, Kang Y, Huo Z, et al. Highly crystalline multimetallic nanoframes with three-dimensional electrocatalytic surfaces. Science, 2014, 343: 1339-1343

9 Braun E, Eichen Y, Sivan U, et al. DNA-templated assembly and electrode attachment of a conducting silver wire. Nature, 1998, 391: $775-778$

$10 \mathrm{Xu} \mathrm{L}$, Jiang Z, Qing Q, et al. Design and synthesis of diverse functional kinked nanowire structures for nanoelectronic bioprobes. Nano Lett, 2012, 13: 746-751

11 Okamoto H, Imura K. Near-field optical imaging of enhanced electric fields and plasmon waves in metal nanostructures. Prog Surf Sci, 2009, 84: 199-229

12 Krogstrup P, Jorgensen HI, Heiss M, et al. Single-nanowire solar cells beyond the shockley-queisser limit. Nat Photon, 2013, 7: 306-310

13 Garcia-Vidal FJ, Pitarke JM, Pendry JB. Silver-filled carbon nanotubes used as spectroscopic enhancers. Phys Rev B, 1998, 58: 6783-6786

14 Pan T, Zang TC, Mao HM, et al. Optical properties of the Au-Ag alloy nanowire coated with an anisotropic shell. Appl Phys A Mater Sci Proc, 2010, 100: 159-164

15 Lan X, Bai J, Masala S, et al. Self-assembled, nanowire network electrodes for depleted bulk heterojunction solar cells. Adv Mater, 2013,
25: $1769-1773$

16 Xia Y, Xiong YJ, Lim B, et al. Shape-controlled synthesis of metal nanocrystals: simple chemistry meets complex physics? Angew Chem Int Edit, 2009, 48: 60-103

17 Cui CH, Yu SH. Engineering interface and surface of noble metal nanoparticle nanotubes toward enhanced catalytic activity for fuel cell applications. Acc Chem Res, 2013, 46: 1427-1437

$18 \mathrm{Li} \mathrm{HH}$, Zhao S, Gong M, et al. Ultrathin PtPdTe nanowires as superior catalysts for methanol electrooxidation. Angew Chem Int Ed, 2013, 52: 7472-7476

19 Xia BY, Wu HB, Yan Y, et al. Ultrathin and ultralong single-crystal platinum nanowire assemblies with highly stable electrocatalytic activity. J Am Chem Soc, 2013, 135: 9480-9485

20 Yogeswaran U, Chen SM. A review on the electrochemical sensors and biosensors composed of nanowires as sensing material. Sensors, 2008, 8: 290-313

21 Sun Y. Silver nanowires-unique templates for functional nanostructures. Nanoscale, 2010, 2: 1626-1642

22 Daniel L, Gaël G, Céline M, et al. Flexible transparent conductive materials based on silver nanowire networks: a review. Nanotechnology, 2013, 24: 452001

23 Huang X, Jain PK, El-Sayed IH, et al. Gold nanoparticles: interesting optical properties and recent applications in cancer diagnostic and therapy. Nanomedicine, 2007, 2: 681-693

24 Jana NR, Gearheart L, Murphy CJ. Seed-mediated growth approach for shape-controlled synthesis of spheroidal and rod-like gold nanoparticles using a surfactant template. Adv Mater, 2001, 13: 1389-1393

25 Sonnichsen C, Franzl T, Wilk T, et al. Drastic reduction of plasmon damping in gold nanorods. Phys Rev Lett, 2002, 88: 077402

26 Nikoobakht B, El-Sayed MA. Preparation and growth mechanism of gold nanorods (NRs) using seed-mediated growth method. Chem Mater, 2003, 15: 1957-1962

27 Shi H, Zhang L, Cai W. Composition modulation of optical absorption in $\mathrm{Ag}_{x} \mathrm{Au}_{1-x}$ alloy nanocrystals in situ formed within pores of mesoporous slica. J Appl Phys, 2000, 87: 1572-1574

28 Hong X, Wang D, Yu R, et al. Ultrathin Au-Ag bimetallic nanowires with Coulomb blockade effects. Chem Comm, 2011, 47: 5160-5162

29 Halder A, Ravishankar N. Ultrafine single-crystalline gold nanowire arrays by oriented attachment. Adv Mater, 2007, 19: 1854-1858

30 Kim JU, Cha SH, Shin K, et al. Preparation of gold nanowires and nanosheets in bulk block copolymer phases under mild conditions. Adv Mater, 2004, 16: 459-464 
31 Barth S, Hernandez-Ramirez F, Holmes JD, et al. Synthesis and applications of one-dimensional semiconductors. Prog Mater Sci, 2010, 55: 563-627

32 Huo ZY, Tsung CK, Huang WY, et al. Sub-two nanometer single crystal Au nanowires. Nano Lett, 2008, 8: 2041-2044

33 Lu XM, Yavuz MS, Tuan HY, et al. Ultrathin gold nanowires can be obtained by reducing polymeric strands of oleylamine- $\mathrm{AuCl}$ complexes formed via aurophilic interaction. J Am Chem Soc, 2008, 130: 8900-8901

34 Pazos-Perez N, Baranov D, Irsen S, et al. Synthesis of flexible, ultrathin gold nanowires in organic media. Langmuir, 2008, 24: 9855-9860

35 Wang C, Hu YJ, Lieber CM, et al. Ultrathin Au nanowires and their transport properties. J Am Chem Soc, 2008, 130: 8902-8903

36 Park HS, Ji CJ. On the thermomechanical deformation of silver shape memory nanowires. Acta Mater, 2006, 54: 2645-2654

37 Velez P, Dassie SA, Leiva EPM. When do nanowires break? A model for the theoretical study of the long-term stability of monoatomic nanowires. Chem Phy Lett, 2008, 460: 261-265

38 Couchman PR, Jesser WA. Thermodynamic theory of size dependence of melting temperature in metals. Nature, 1977, 269: 481-483

39 Link S, Burda C, Mohamed MB, et al. Laser photothermal melting and fragmentation of gold nanorods: energy and laser pulse-width dependence. J Phys Chem A, 1999, 103: 1165-1170

40 Link S, El-Sayed MA. Spectroscopic determination of the melting energy of a gold nanorod. J Chem Phys, 2001, 114: 2362-2368

41 Plech A, Kotaidis V, Gresillon S, et al. Laser-induced heating and melting of gold nanoparticles studied by time-resolved X-ray scattering. Phys Rev B, 2004, 70: 195423

42 Petrova H, Juste JP, Pastoriza-Santos I, et al. On the temperature stability of gold nanorods: comparison between thermal and ultrafast laser-induced heating. Phys Chem Chem Phys, 2006, 8: 814-821

43 Wang YT, Teitel S, Dellago C. Surface-driven bulk reorganization of gold nanorods. Nano Lett, 2005, 5: 2174-2178

44 Bai XM, Li M. Nucleation and melting from nanovoids. Nano Lett, 2006, 6: 2284-2289

45 de Bas BS, Ford MJ, Cortie MB. Melting in small gold clusters: a density functional molecular dynamics study. J Phys Condens Matter, 2006, 18: 55-74

46 Weeber JC, Dereux A, Girard C, et al. Plasmon polaritons of metallic nanowires for controlling submicron propagation of light. Phys Rev B, 1999, 60: 9061-9068

47 Koga K, Ikeshoji T, Sugawara K. Size- and temperature-dependent structural transitions in gold nanoparticles. Phys Rev Lett, 2004, 92 115507

48 Karim S, Toimil-Molares ME, Balogh AG, et al. Morphological evolution of Au nanowires controlled by Rayleigh instability. Nano- technology, 2006, 17: 5954

49 Kang YJ, Ye XC, Murray CB. Size- and shape-selective synthesis of metal nanocrystals and nanowires using $\mathrm{CO}$ as a reducing agent. Angew Chem Int Ed, 2010, 49: 6156-6159

50 Wu J, Gross A, Yang H. Shape and composition-controlled platinum alloy nanocrystals using carbon monoxide as reducing agent. Nano Lett, 2011, 11: 798-802

51 Liu K, Zhao N, Kumacheva E. Self-assembly of inorganic nanorods. Chem Soc Rev, 2011, 40: 656-671

52 Link S, Wang ZL, El-Sayed MA. How does a gold nanorod melt? J Phys Chem B, 2000, 104: 7867-7870

53 Eichler A. CO oxidation on transition metal surfaces: reaction rates from first principles. Surf Sci, 2002, 498: 314-320

54 Somorjai GA, Li Y. Introduction to Surface Chemistry and Catalysis. Hoboken: John Wiley \& Sons, 2010: 437-480

55 Zhang CJ, Hu P. CO oxidation on Pd(100) and Pd(111): a comparative study of reaction pathways and reactivity at low and medium coverages. J Am Chem Soc, 2001, 123: 1166-1172

56 Liu QS, Yan Z, Henderson NL, et al. Synthesis of CuPt nanorod catalysts with tunable lengths. J Am Chem Soc, 2009, 131: 5720-5721

57 Yu W, Porosoff MD, Chen JG. Review of Pt-based bimetallic catalysis: from model surfaces to supported catalysts. Chem Rev, 2012, 112: 5780-5817

58 Link S, El-Sayed MA. Spectral properties and relaxation dynamics of surface plasmon electronic oscillations in gold and silver nanodots and nanorods. J Phys Chem B, 1999, 103: 8410-8426

59 Takahata R, Yamazoe S, Koyasu K, et al. Surface plasmon resonance in gold ultrathin nanorods and nanowires. J Am Chem Soc, 2014, 136: 8489-8491

Acknowledgements This work was supported in part by the National Science Foundation of USA (CHE-1213926). In situ TEM study was carried out at the Center for Functional Nanomaterials, Brookhaven National Laboratory, which is supported by the USA Department of Energy, Office of Basic Energy Sciences, under Contract No. DE-SC0012704. Wu J is grateful for Shen Fellowship from UIUC. We thank Rajinder Singh and Nitin Tyagi for carrying out the experiments on the preparation of $\mathrm{AgAu}$ nanowires.

Author contributions Yang $\mathrm{H}$ and $\mathrm{Wu} \mathrm{J}$ conceived the study and wrote the manuscript. Su D executed the in-situ variable temperature TEM study. Pan YT acquired and analyzed the absorption spectra. All authors contributed to the discussion.

Conflict of interest The authors declare that they have no conflict of interest.

Supplementary information Experimental details and supporting data are available in the online version of the paper. 


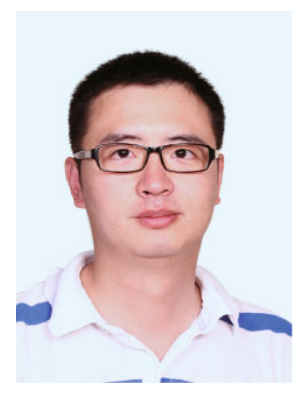

Jianbo Wu was born in Zhejiang, China, in 1982. He received his BSc degree in 2005, and MSc degree in 2007, both from Zhejiang University. He received his PhD degree majored in chemical engineering in 2012 under the direction of Prof. Hong Yang at University of Rochester, and then did his postdoc research jointly in Prof. Hong Yang (2012-2014) and Prof. Jian-Min Zuo (2013-2014) groups at the University of Illinois at Urbana-Champaign (UIUC). He received Elon Huntington Hooker Fellowship from the University of Rochester in 2011, and Shen Postdoctoral Fellowship from Chemical and Biomolecular Engineering Department, UIUC in 2013-2014. He was recruited by Shanghai Jiaotong University under the "National Thousand Talents Plan for Young Professionals" in 2014. His research interest is on colloidal chemistry, shape control of platinum-based alloys and intermetallics, and their applications as electrocatalysts.

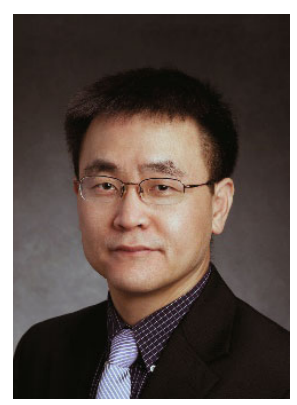

Hong Yang is a Richard C Alkire Professor of Chemical and Biomolecular Engineering at UIUC. He received his BSc degree from Tsinghua University (1989), MSc degree from the University of Victoria (1994), and PhD degree from the University of Toronto (1998). After working at Harvard University as an NSERC postdoctoral fellow (1998-2001), he started his career at the University of Rochester before joining the Department of Chemical and Biomolecular Engineering at UIUC in 2012. Dr. Yang is an NSERC Canada Doctoral Prize winner and an NSF CAREER Award recipient. His current research interests include control of nanocrystals, surface modification, in situ TEM study of nanomaterials in solution and under reacting atmosphere, catalysis and electrocatalysis, and nanomaterials for energy and biological applications.

中文摘要 本文通过一氧化碳辅助GRAILS法 (gas reducing agent in liquid solution)制备得到稳定的亚 $2 \mathrm{~nm}$ 粗细的银金合金纳米线. 这 些银金合金纳米线在加热和在电子束辐照的条件下表现出良好的稳定性. 对其纳米结构进行表征, 我们发现这种纳米线沿纳米线径向 主要由 $\{111\}$ 晶面组成. 这个结构不同于没有一氧化碳辅助生长的以 $\{100\}$ 面为主的金纳米线. 后者在电子束辐照的条件下非常不稳定. 结构和成分上的区别导致了稳定性的提高. 